\title{
Spin Order and Phase Transitions in Chains of Polariton Condensates
}

\author{
H. Ohadi, ${ }^{1, *}$ A. J. Ramsay, ${ }^{2}$ H. Sigurdsson, ${ }^{3}$ Y. del Valle-Inclan Redondo, ${ }^{1}$ S. I. Tsintzos,${ }^{4}$ Z. Hatzopoulos, ${ }^{4}$ \\ T. C. H. Liew, ${ }^{5}$ I. A. Shelykh, ${ }^{3,6}$ Y. G. Rubo, ${ }^{7,8}$ P. G. Savvidis, ${ }^{4,6,9}$ and J. J. Baumberg ${ }^{1, \dagger}$ \\ ${ }^{1}$ NanoPhotonics Centre, Department of Physics, Cavendish Laboratory, University of Cambridge, \\ Cambridge CB3 OHE, United Kingdom \\ ${ }^{2}$ Hitachi Cambridge Laboratory, Hitachi Europe Ltd., Cambridge CB3 OHE, United Kingdom \\ ${ }^{3}$ Science Institute, University of Iceland, Dunhagi-3, IS-107 Reykjavik, Iceland \\ ${ }^{4}$ FORTH, Institute of Electronic Structure and Laser, 71110 Heraklion, Crete, Greece \\ ${ }^{5}$ School of Physical and Mathematical Sciences, Nanyang Technological University 637371, Singapore \\ ${ }^{6}$ ITMO University, St. Petersburg 197101, Russia \\ ${ }^{7}$ Instituto de Energías Renovables, Universidad Nacional Autónoma de México, Temixco, Morelos, 62580, Mexico \\ ${ }^{8}$ Center for Theoretical Physics of Complex Systems, Institute for Basic Science (IBS), Daejeon 34051, Republic of Korea \\ ${ }^{9}$ Department of Materials Science and Technology, University of Crete, 71003 Heraklion, Crete, Greece
}

(Received 2 March 2017; published 8 August 2017)

\begin{abstract}
We demonstrate that multiply coupled spinor polariton condensates can be optically tuned through a sequence of spin-ordered phases by changing the coupling strength between nearest neighbors. For closed four-condensate chains these phases span from ferromagnetic (FM) to antiferromagnetic (AFM), separated by an unexpected crossover phase. This crossover phase is composed of alternating FM-AFM bonds. For larger eight-condensate chains, we show the critical role of spatial inhomogeneities and demonstrate a scheme to overcome them and prepare any desired spin state. Our observations thus demonstrate a fully controllable nonequilibrium spin lattice.
\end{abstract}

DOI: 10.1103/PhysRevLett.119.067401

Spin models, such as the Ising model, have been very successful in describing a wide range of condensed matter phenomena [1]. In addition, these models can be mapped to real-world optimization problems [2,3], for example, in transport scheduling, artificial intelligence, and financial portfolio optimization [4,5]. Consequently, there is a growing interest in building controlled spin lattices both to study computationally complex spin systems such as spin glasses [6], but also as a potential computing architecture $[7,8]$. Several systems have been explored, including ultracold atoms [9], degenerate optical parametric oscillators [10,11], electromechanical resonators [12], and CMOS transistors $[4,5,13]$. Recently, individual exciton-polariton (polariton) condensates [14-21] have been observed to spontaneously magnetize [22], and when two condensates are close together the spins can be controllably aligned (or antialigned) [23]. Using these building blocks, we now explore the scaling up to a large 1D system, constructing a nonequilibrium, driven-dissipative controlled spin lattice of exciton-polariton condensates. New types of order can appear in larger lattices, while at the same time extra measures have to be taken to ensure scalability.

Here, we study the spin properties of a closed interacting chain of exciton-polariton condensates. When the pump laser is turned on, the system spontaneously condenses into a magnetically ordered state on picosecond time scales, and remains frozen in that state for many seconds. By optically tuning the Josephson coupling between the condensates, the system can be tuned from a ferromagnetic to antiferromagnetic phase, via a disordered crossover phase. Remarkably, in a system of 4 identical spin condensates, where there is no spatial disorder, a paired spin state with alternating FM-AFM bonds is observed. Such a state cannot exist in a smaller system. Furthermore, despite the larger phase degree of freedom offered by the larger spin chain, from comparison to theory we conclude that the FM (AFM) bonds only adopt a phase shift of $0(\pi)$, respectively. This locking of the phase and spin effectively results in a binary spin system. As the system size is increased to longer condensate chains, spatial inhomogeneity in the microcavity becomes an issue. We demonstrate a strategy to engineer ferromagnetic, antiferromagnetic, or glassy states of longer spin chains by simultaneously tailoring each individual nearest neighbor (NN) coupling between sites. Our work introduces interacting trapped polariton condensates as a controllable system for studying complex nonlinear spin models out of equilibrium.

Polaritons are mixed light-matter quasiparticles appearing due to the strong coupling of photons in a microcavity and excitons in a semiconductor quantum well [24]. Polaritons are driven-dissipative bosons that can condense into macroscopically coherent many-body states [14-17]. High optical accessibility, picosecond dynamics, large nonlinearity [25] and other unique properties [26-32], with potential application in semiconductor chip devices [18,19,33-37] make them particularly attractive.

Our system is a GaAs quantum-well microcavity (see Supplemental Material [38], 1) with an optically induced 
(a)

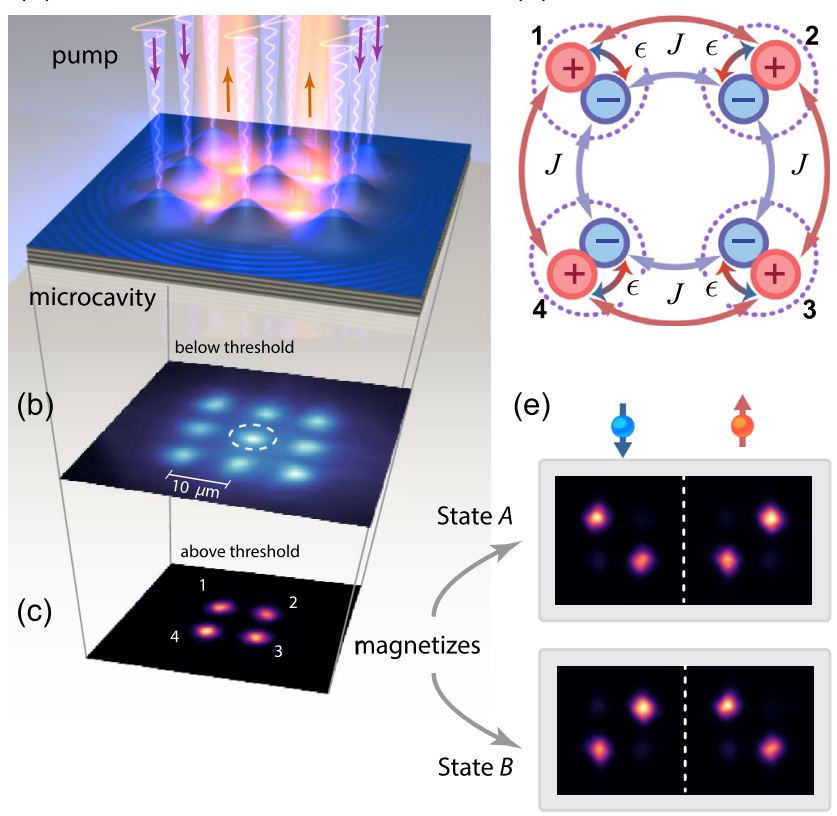

FIG. 1. (a) Lattice potential in the microcavity created by blueshifts at the pump beams (purple beams) forming magnetized condensates in the center of each site (yellow spots). (b) Below threshold PL showing the pump spots. Global NN barrier is tuned by modulating the intensity of the center spot $\left(u_{r}\right.$, dashed circle). (c) Formation of condensates at the center of each of the 4 lattice sites above threshold. (d) Schematic of condensate spin chain comprised of two coupled Bose-Hubbard chains. Each condensate (indices 1-4) has two spin states (+ and - ), which are coherently coupled by $\epsilon$. Each is also coherently coupled to its same spin NN by Josephson coupling $J$. (e) Magnetization of condensate chain (expt) above the spin-bifurcation threshold $\left(P_{\mathrm{c}}\right)$, into an AFM state.

two-dimensional square lattice potential where a magnetized polariton condensate (emitting almost fully circularly polarized light) forms at each lattice site [Figs. 1(a)-1(c)]. We generate polaritons by the nonresonant optical excitation of the microcavity. Each nonresonantly pumped spot creates a local reservoir of hot excitons which rapidly lose energy and flow out due to repulsion from hot excitons in the reservoir and repulsive self-interactions. Therefore, the optical excitation acts as both the gain and the trapping potential forming the lattice sites $[39,40]$. Polaritons scatter into the ground state by stimulated scattering and final-state bosonic amplification [25]. Once the density at any site surpasses a threshold, a macroscopically coherent condensate forms inside each trap [30,41].

The total spin of the polaritons is quantized along the structure growth axis, which corresponds to right- and leftcircularly polarized photons emitted from the cavity. For pump powers exceeding a spin-bifurcation threshold, trapped polaritons can spontaneously magnetize by condensing into a single, randomly chosen spin state. The spin-bifurcation process is driven by the dissipation rate difference $(\gamma)$ and energy splitting of the horizontally and vertically polarized polaritons $(\epsilon)$, which determine the spin-bifurcation threshold [22]. We operate above this threshold, which means each condensate spontaneously forms in the spin-up or spin-down state with a degree of circular polarization (condensate spin) $\left|S_{z}\right|>85 \%$. Polariton condensates created by the excitation pattern shown in Fig. 1(b) form a closed chain because the potential from the central pump spot is so large that the tunneling of polaritons between diagonal sites is negligible. Therefore, only the nearest neighbor coupling is significant. In this geometry the spins of each condensate are on-site coherently coupled by $\epsilon$, and each is coherently coupled to that of the neighboring condensate by $J$ [Fig. 1(d)]. By varying the power of the central pump spot [Fig. 1(b)] we tune $J$, and by changing the relative ratio of $J / \epsilon$ we can change the magnetic order of the chain. Remarkably, the condensate chains exhibit distinct magnetic phases and mostly align their spins into particular patterns depending on their coupling strengths. Initially we explore a fourcondensate system, before showing how this behavior develops in the eight-condensate version.

Magnetized condensation at the minima of the optically induced lattice potential is seen in the real-space photoluminescence (PL) from below to above the condensation threshold [Fig. 1(e)]. The critical magnetization threshold is 1.3 times the condensation threshold. We denote the intensity of the central pump spot relative to the rest of the lattice spots by $u_{r}$. Since the local blueshift generated by the pump is linearly proportional to the intensity of the pump spots, the barrier height between each neighboring condensate increases as $u_{r}$ increases. Thus, $u_{r}$ is a measure of the coupling strength (Josephson tunneling rate $J$ ) of the condensate lattice; i.e., increasing $u_{r}$ corresponds to decreasing $J$. Since each condensate can form in the spin-up or the spindown states and couples with its two neighboring condensates, we expect to see the formation of different spin patterns around the chain as we tune $u_{r}$.

We observe four distinct phases of the spin chain as we increase $u_{r}$ (Fig. 2): (i) FM with two spin degenerate states, formed from all spin-up or spin-down states, (ii) paired ferromagnetic (PFM) separated by two domain walls, with four possible spin degenerate states and zero total spin, (iii) AFM with two possible spin degenerate states, and (iv) paramagnetism with nearly zero spin correlations between condensates (see also Supplemental Material [38], 2). In each case, the spin chain spontaneously collapses into any of the degenerate states due to random spin fluctuations from the reservoir at the onset of magnetization. Although the final state of the chain is indeterminate for each realization, once the spin chain forms it stays in that particular steady state if a longer pump pulse (e.g., $100 \mathrm{~ms}$ ) is applied.

To characterize the spin correlations in each phase, we calculate the $4 \times 4$ correlation matrix $C$ where the elements $C_{m n}=\rho\left(S_{z, m}, S_{z, n}\right)$ are the Pearson correlation of spins of condensates $m$ and $n$ [as labeled in Figs. 1(c), 1(d)]. 


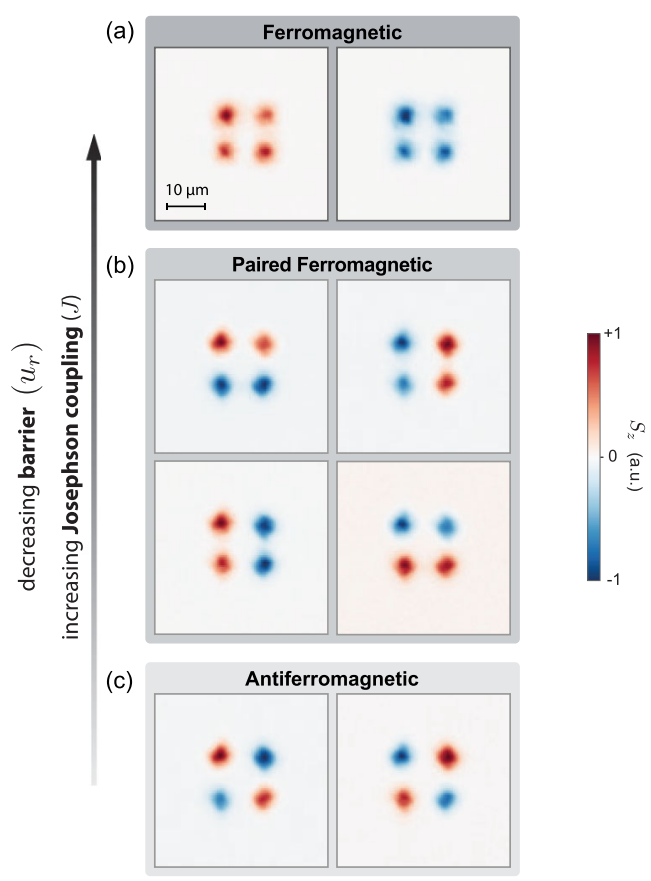

FIG. 2. Steady states as a function of barrier height. The measured condensate spin $S_{z}$ for all possible stable states at three phases of (a) FM, (b) PFM, and (c) AFM, when the global $\mathrm{NN}$ barrier $u_{r}$ is increased.

The correlation matrices for 100 realizations in the AFM and PFM regimes [Figs. 3(a), 3(b)] demonstrate robustly correlated spin chains. To build their phase diagram we plot the average diagonal $\bar{C}_{\text {diag }}=\left(C_{13}+C_{24}\right) / 2$ and side $\bar{C}_{\text {side }}=\left(C_{12}+C_{23}+C_{34}+C_{41}\right) / 4$ condensate spin correlations as a function of $u_{r}$ [Fig. 3(c)]. We observe the FM phase for $u_{r}<0.9$ followed by a sharp and narrow crossover to PFM for $0.9<u_{r}<0.96$ and a second sharp crossover to a broad AFM phase for $0.96<u_{r}<1.1$, succeeded by a rapid decay of correlations to near zero at higher $u_{r}$. 2D Ginzburg-Landau numerical simulations (see the Supplemental Material [38], 4) accurately reproduce the experimental phase map [Fig. 3(d)].

We can easily extend the square pumping geometry to accommodate longer spin chains forming now a total of 8 condensates [Figs. 4(a), 4(b)]. Once again, we observe FM, AFM, and a variety of spin glass states in this magnetic chain. Because the number of barriers to modulate increases, their simultaneous control is not as straightforward. At the same time, as the system size increases, tiny spatial inhomogeneities in the microcavity become increasingly important. The latter arise from the growth process and slightly change the local energy of the polaritons, modulating the coupling strength between neighboring sites. If the energy modulation is large enough, it can even change the type of the coupling at each bond. Without more sophisticated approaches, this spatial inhomogeneity of the microcavity would limit the size of condensate lattices that can be studied, and thus prospects for using the system as a simulator. This general issue is, however, generic in all condensate lattices.

We can, however, explore and correct for the spatial inhomogeneity here by tailoring the imprinted excitation pattern. Since the background energy landscape is unknown, we employ an iterative search algorithm with feedback to find the optimal pattern needed to produce a desired correlated spin chain (see the Supplemental Material [38], 3). At the end of each search process, which only takes a few minutes, the most likely spin states can be inspected [Fig. 4(c)]. Principal component analysis (PCA) of the spin-up and spin-down intensities reveals the most probable states after optimization [Fig. 4(d)]. In the FM and AFM phases, the pure states (1st PCA components) are obtained in $60 \%$ of instances, more than twice as likely as trapping a single defect (2nd PCA component) with two domain walls. Other states have $<10 \%$ probabilities. By contrast, in the glass state we find near-degenerate states with four domain walls that dominate. Our twodimensional (2D) simulations show that a disorder potential of $\sim 5 \mu \mathrm{eV}$ is enough to break spin chain symmetry (see the
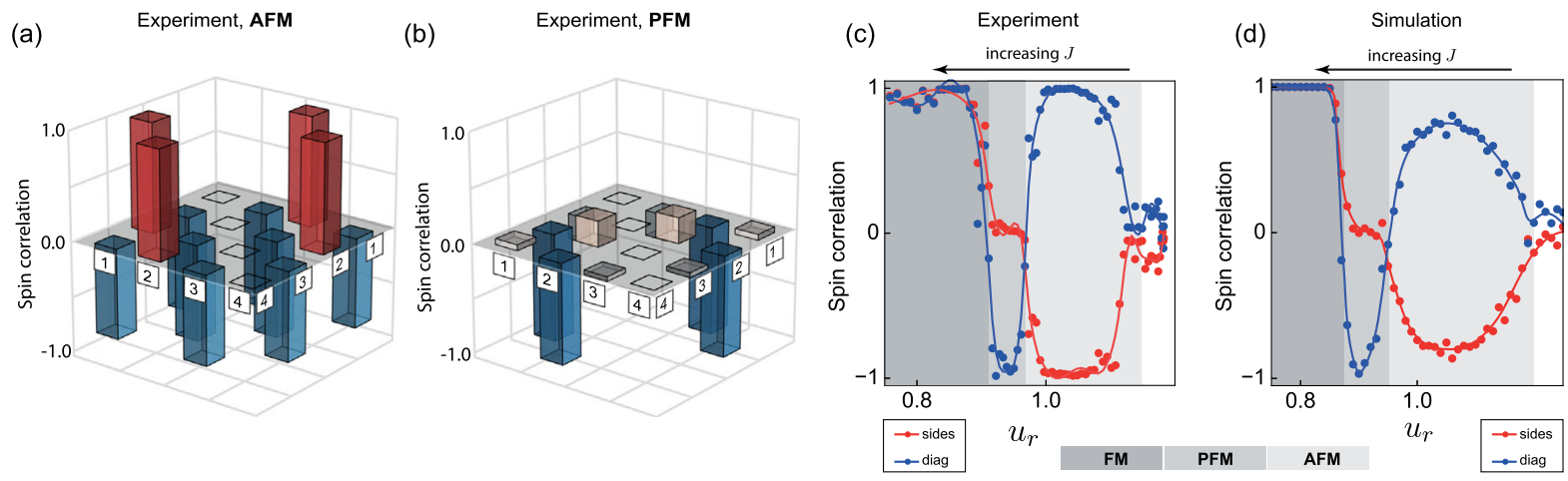

FIG. 3. Spin correlations as a function of barrier height. (a), (b) The measured correlation matrix of the spin chain at the two phases of (a) AFM and (b) PFM. Numbers show condensate indices. The autocorrelated diagonal elements are removed for clarity. (c), (d) Phase diagram of the spin chain showing spin correlation of the diagonal and side condensates vs $u_{r}$ in (c) experiment and (d) 2D numerical simulations. 


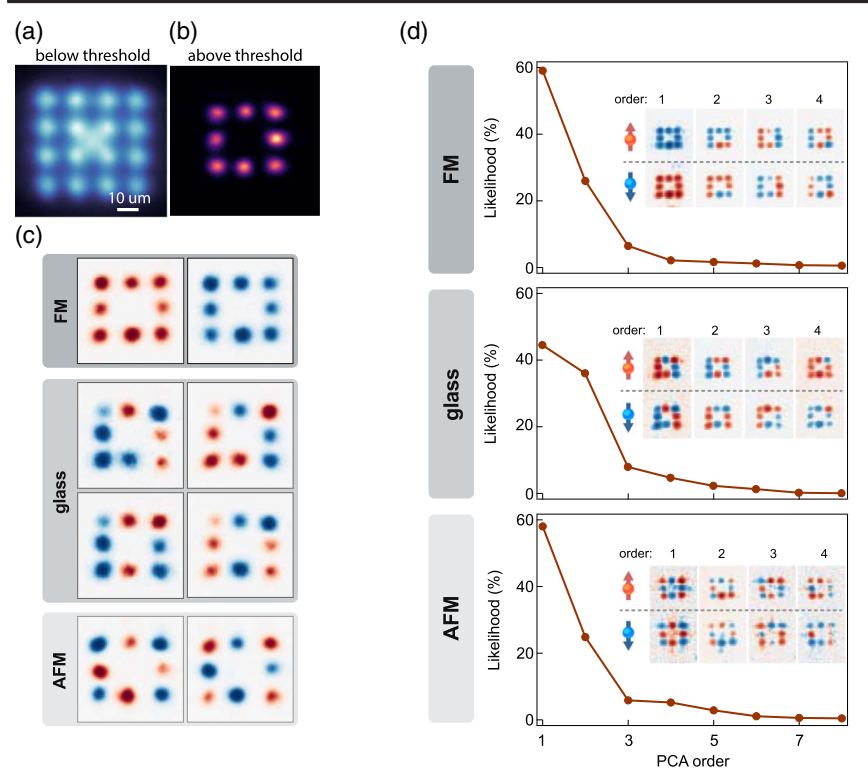

FIG. 4. Controlling magnetic order with feedback. (a) PL below threshold showing excitation pattern. (b) Emission above threshold showing eight-condensate chain. (c) The most probable spin states after optimization using FM, glass, or AFM search criteria in (d). (d) PCA components of spin-up or spin-down emission after the search procedure for different targets. Intensities show strengths of components. Plots show likelihood (variance percentage) for each order.

Supplemental Material [38], 4). We thus show this method can initialize the spin chain in any desired state.

We outline here a mean-field theory, extended from single trapped condensates [22,42] to include the Josephson coupling between nearest neighbors [23]. The order parameter for each exciton-polariton condensate is a two-component complex vector $\Psi_{n}=\left[\psi_{n+}, \psi_{n-}\right]^{\mathrm{T}}$, where $\psi_{n+}$ and $\psi_{n-}$ are the spin-up and spin-down wave functions of condensate $n$. The order parameters evolve according to the driven dissipative equation:

$$
\begin{aligned}
i \dot{\Psi}_{n}= & -\frac{i}{2} g\left(S_{n}\right) \Psi_{n}-\frac{i}{2}(\gamma-i \epsilon) \sigma_{x} \Psi_{n} \\
& +\frac{1}{2}\left(\bar{\alpha} S_{n}+\alpha S_{n z} \sigma_{z}\right) \Psi_{n} \quad \text { (interactions) } \\
& -\frac{J}{2}\left(\Psi_{n-1}+\Psi_{n+1}\right) \quad \text { (Josephson coupling) }
\end{aligned}
$$

Here, $g\left(S_{n}\right)=\Gamma-W+\eta S_{n}$ is the pumping-dissipation balance, $\Gamma$ is the (average) dissipation rate, $W$ is the incoherent in-scattering, $S_{n}=\left(\left|\psi_{n-}\right|^{2}+\left|\psi_{n+}\right|^{2}\right) / 2$, and $\eta$ captures the gain-saturation term [43]. Linearly polarized single-polariton states in $X$ (horizontal) and $Y$ (vertical) are split in energy by $\epsilon$ and in dissipation rate by $\gamma$, and $\sigma_{x, z}$ are the Pauli matrices. The nonlinear interaction constants are given by $\bar{\alpha}=\alpha_{1}+\alpha_{2}$ and $\alpha=\alpha_{1}-\alpha_{2}$, where $\alpha_{1}$ is the interaction constant for polaritons with the same spin and $\alpha_{2}$ is the interaction constant for polaritons with opposite spins.
Finally, $J>0$ is the spin-preserving Josephson coupling [44-46] between nearest-neighbor condensates.

By making an ansatz where FM (AFM) bonds have a relative phase of $0(\pi)$ between nearest neighbors, we construct a mean-field model (see the Supplemental Material [38], 5). This maps the system to a single condensate with an energy shift $\omega_{J}$ and a renormalized polarization splitting $\epsilon_{J}$. This allows us to apply the findings of Ref. [22] for a single condensate to explain the phase diagram of Figs. 3(c), 3(d) using two criteria: (i) the final state must be stable, and (ii) if multiple states are stable, then the most probable final state is the one which turns magnetic at the lowest power. To be stable requires $\epsilon_{J}>0$, so that onsite spin coupling is strong enough to give magnetized condensates. In addition, the spin-bifurcation threshold favors states with low $\epsilon_{J}$ (see Eqs. S5 and S6, [38]). The three most favorable spin phases then yield modified splittings: $\epsilon_{J}^{\mathrm{FM}}=\epsilon, \epsilon_{J}^{\mathrm{PFM}}=\epsilon-J, \epsilon_{J}^{\mathrm{AFM}}=\epsilon-2 J$. Hence, the phase diagram of Fig. 3(c) is explained as follows. For $J<\epsilon / 2$, all three states are stable but the AFM state is favored since it has the lowest $\epsilon_{J}$. For $\epsilon / 2<J<\epsilon$, the AFM state becomes unstable and the glass state is selected since it is now the lowest threshold state. For $J>\epsilon$, only the FM state is stable. This explains all the key behaviors observed.

In conclusion, we demonstrate control of the spin states of closed chains of four- and eight-polariton condensates. For small chains, the nonequilibrium driven-dissipative spin lattice gives rise to a unique paired spin (paired-FM) ordered state. This observation shows that our system is not governed by the minimization of free-energy, as in, for example, the standard equilibrium Ising model. To our knowledge, this paired-FM phase has not been observed in any equilibrium or nonequilibrium binary spin system. In a 2D square lattice, in the paired-FM phase each site must have two FM and two AFM bonds. Realizations of this phase can be mapped to different tilings of a chessboard with dominoes, which is a $\# P$-complete problem [47]. We find that sample inhomogeneity hinders straightforward scaling to larger chains. We overcome this problem by careful feedback algorithms that compensate for sample inhomogeneities and demonstrate a proof-of-principle scaling method. In the absence of any corrections, the system behaves like spin glass, where interactions are randomly chosen by the sample inhomogeneities acting as "quenched disorder."

The raw data for this work is available at Ref. [48].

We acknowledge Grants No. EPSRC EP/L027151/1, No. EU INDEX 289968, No. ERC "POLAFLOW" Starting Grant, ERC LINASS 320503, Spanish MEC (MAT200801555), Mexican CONACYT 251808, Leverhulme Trust Grant No. VP1-2013-011 and Fundación La Caixa. H. S. and I. S. acknowledge support by the Research Fund of the University of Iceland, The Icelandic Research Fund, Grant No. 163082-051. T. L. was supported by the MOE AcRF Tier 1 Grant No. 2016-T1-001-084. P. S. acknowledges financial support from the Stavros Niarchos Foundation, "ARCHERS" project. 
*ho278@cam.ac.uk

†jb12@cam.ac.uk

[1] R. J. Baxter, Exactly Solved Models in Statistical Mechanics (Elsevier, New York, 1989).

[2] S. Kirkpatrick, C. D. Gelatt, and M. P. Vecchi, Science 220, 671 (1983).

[3] A. Lucas, Front. Phys. 2, 5 (2014).

[4] M. Hayashi, M. Yamaoka, C. Yoshimura, T. Okuyama, H. Aoki, and H. Mizuno, Int. J. Net. Comp. 6, 195 (2016).

[5] M. Yamaoka, C. Yoshimura, M. Hayashi, T. Okuyama, H. Aoki, and H. Mizuno, Hitachi Review 65, 156 (2016).

[6] F. Barahona, J. Phys. A 15, 3241 (1982).

[7] I. Buluta and F. Nori, Science 326, 108 (2009).

[8] I. M. Georgescu, S. Ashhab, and F. Nori, Rev. Mod. Phys. 86, 153 (2014).

[9] I. Bloch, J. Dalibard, and S. Nascimbène, Nat. Phys. 8, 267 (2012).

[10] A. Marandi, Z. Wang, K. Takata, R. L. Byer, and Y. Yamamoto, Nat. Photonics 8, 937 (2014).

[11] T. Inagaki, K. Inaba, R. Hamerly, K. Inoue, Y. Yamamoto, and H. Takesue, Nat. Photonics 10, 415 (2016).

[12] I. Mahboob, H. Okamoto, and H. Yamaguchi, Sci. Adv. 2, e1600236 (2016).

[13] M. Taiji, N. Ito, and M. Suzuki, Rev. Sci. Instrum. 59, 2483 (1988).

[14] H. Deng, G. Weihs, C. Santori, J. Bloch, and Y. Yamamoto, Science 298, 199 (2002).

[15] J. Kasprzak, M. Richard, S. Kundermann, A. Baas, P. Jeambrun, J. M. J. Keeling, F. M. Marchetti, M. H. Szymańska, R. André, J. L. Staehli et al., Nature (London) 443, 409 (2006).

[16] R. Balili, V. Hartwell, D. Snoke, L. Pfeiffer, and K. West, Science 316, 1007 (2007).

[17] J. Baumberg, A. Kavokin, S. Christopoulos, A. Grundy, R. Butté, G. Christmann, D. Solnyshkov, G. Malpuech, G. Baldassarri Höger von Högersthal, E. Feltin et al., Phys. Rev. Lett. 101, 136409 (2008).

[18] C. Schneider, A. Rahimi-Iman, N. Y. Kim, J. Fischer, I. G. Savenko, M. Amthor, M. Lermer, A. Wolf, L. Worschech, V. D. Kulakovskii, I. A. Shelykh, M. Kamp, S. Reitzenstein, A. Forchel, Y. Yamamoto, and S. Höfling, Nature (London) 497, 348 (2013).

[19] P. Bhattacharya, B. Xiao, A. Das, S. Bhowmick, and J. Heo, Phys. Rev. Lett. 110, 206403 (2013).

[20] K. S. Daskalakis, S. A. Maier, R. Murray, and S. Kéna-Cohen, Nat. Mater. 13, 271 (2014).

[21] J. D. Plumhof, T. Stöferle, L. Mai, U. Scherf, and R. F. Mahrt, Nat. Mater. 13, 247 (2014).

[22] H. Ohadi, A. Dreismann, Y. G. Rubo, F. Pinsker, Y. del Valle-Inclan Redondo, S. I. Tsintzos, Z. Hatzopoulos, P. G. Savvidis, and J. J. Baumberg, Phys. Rev. X 5, 031002 (2015).

[23] H. Ohadi, Y. del Valle-Inclan Redondo, A. Dreismann, Y. G. Rubo, F. Pinsker, S. I. Tsintzos, Z. Hatzopoulos, P. G. Savvidis, and J. J. Baumberg, Phys. Rev. Lett. 116, 106403 (2016).

[24] A. V. Kavokin, J. Baumberg, G. Malpuech, and F. P. Laussy, Microcavities (Oxford University Press, Oxford, 2007).

[25] P. G. Savvidis, J. J. Baumberg, R. M. Stevenson, M. S. Skolnick, D. M. Whittaker, and J. S. Roberts, Phys. Rev. Lett. 84, 1547 (2000).
[26] C. Leyder, M. Romanelli, J. P. Karr, E. Giacobino, T. C. H. Liew, M. M. Glazov, A. V. Kavokin, G. Malpuech, and A. Bramati, Nat. Phys. 3, 628 (2007).

[27] K. G. Lagoudakis, T. Ostatnický, A. V. Kavokin, Y. G. Rubo, R. André, and B. Deveaud-Plédran, Science 326, 974 (2009).

[28] T. K. Paraïso, M. Wouters, Y. Léger, F. Morier-Genoud, and B. Deveaud-Plédran, Nat. Mater. 9, 655 (2010).

[29] M. Abbarchi, A. Amo, V. G. Sala, D. D. Solnyshkov, H. Flayac, L. Ferrier, I. Sagnes, E. Galopin, A. Lemaître, G. Malpuech, and J. Bloch, Nat. Phys. 9, 275 (2013).

[30] P. Cristofolini, A. Dreismann, G. Christmann, G. Franchetti, N. G. Berloff, P. Tsotsis, Z. Hatzopoulos, P. G. Savvidis, and J. J. Baumberg, Phys. Rev. Lett. 110, 186403 (2013).

[31] V. G. Sala, D. D. Solnyshkov, I. Carusotto, T. Jacqmin, A. Lemaître, H. Terças, A. Nalitov, M. Abbarchi, E. Galopin, I. Sagnes et al., Phys. Rev. X 5, 011034 (2015).

[32] S. Dufferwiel, F. Li, E. Cancellieri, L. Giriunas, A. A. P. Trichet, D. M. Whittaker, P. M. Walker, F. Fras, E. Clarke, J. M. Smith, M. S. Skolnick, and D. N. Krizhanovskii, Phys. Rev. Lett. 115, 246401 (2015).

[33] A. Amo, T. C. H. Liew, C. Adrados, R. Houdre, E. Giacobino, A. V. Kavokin, and A. Bramati, Nat. Photonics 4, 361 (2010).

[34] D. Ballarini, M. De Giorgi, E. Cancellieri, R. Houdré, E. Giacobino, R. Cingolani, A. Bramati, G. Gigli, and D. Sanvitto, Nat. Commun. 4, 1778 (2013).

[35] R. Cerna, Y. Léger, T. K. Paraïso, M. Wouters, F. Morier-Genoud, M. T. Portella-Oberli, and B. Deveaud, Nat. Commun. 4, 2008 (2013).

[36] H. S. Nguyen, D. Vishnevsky, C. Sturm, D. Tanese, D. Solnyshkov, E. Galopin, A. Lemaître, I. Sagnes, A. Amo, G. Malpuech, and J. Bloch, Phys. Rev. Lett. 110, 236601 (2013).

[37] A. Dreismann, H. Ohadi, Y. del Valle-Inclan Redondo, R. Balili, Y. G. Rubo, S. I. Tsintzos, G. Deligeorgis, Z. Hatzopoulos, P. G. Savvidis, and J. J. Baumberg, Nat. Mater. 15, 1074 (2016).

[38] See Supplemental Material at http://link.aps.org/ supplemental/10.1103/PhysRevLett.119.067401 for more details of experiment, theoretical model and simulations.

[39] E. Wertz, L. Ferrier, D. D. Solnyshkov, R. Johne, D. Sanvitto, A. Lemaítre, I. Sagnes, R. Grousson, A. V. Kavokin, P. Senellart, G. Malpuech, and J. Bloch, Nat. Phys. 6, 860 (2010).

[40] G. Tosi, G. Christmann, N. G. Berloff, P. Tsotsis, T. Gao, Z. Hatzopoulos, P. G. Savvidis, and J. J. Baumberg, Nat. Phys. 8, 190 (2012).

[41] A. Askitopoulos, H. Ohadi, A. V. Kavokin, Z. Hatzopoulos, P. G. Savvidis, and P. G. Lagoudakis, Phys. Rev. B 88, 041308 (2013).

[42] I. L. Aleiner, B. L. Altshuler, and Y. G. Rubo, Phys. Rev. B 85, 121301 (2012).

[43] J. Keeling and N. G. Berloff, Phys. Rev. Lett. 100, 250401 (2008).

[44] K. G. Lagoudakis, B. Pietka, M. Wouters, R. André, and B. Deveaud-Plédran, Phys. Rev. Lett. 105, 120403 (2010).

[45] M. Wouters, Phys. Rev. B 77, 121302 (2008).

[46] M. O. Borgh, J. Keeling, and N. G. Berloff, Phys. Rev. B 81, 235302 (2010).

[47] J. Matousek, Thirty-Three Miniatures: Mathematical and Algorithmic Applications of Linear Algebra (American Mathematical Society, Providence, RI, 2010), Chap. 22.

[48] DOI: 10.17863/CAM.11959. 